This document is the accepted manuscript version of the following article: Engelstädter, J., Sandrock, C., \& Vorburger, C. (2011). Contagious parthenogenesis, automixis, and a sex determination meltdown. Evolution, International Journal of Organic Evolution, 65(2), 501-511. http://doi.org/10.1111/j.1558-5646.2010.01145.x

\title{
Contagious parthenogenesis, automixis, and a sex
}

\section{determination meltdown}

Jan Engelstädter ${ }^{1,2, *}$, Christoph Sandrock ${ }^{3,4,5} \&$ Christoph Vorburger ${ }^{1,6,7}$

${ }^{1}$ Institute of Integrative Biology, ETH Zurich, Universitätsstr.16, 8092 Zurich,
Switzerland

${ }^{2}$ E-mail: jan.engelstaedter@env.ethz.ch

${ }^{3}$ Institute of Evolutionary Biology and Environmental Studies, Winterthurerstrasse 190, 8057 Zürich, University of Zurich, Switzerland

${ }^{4}$ present address: Swiss Bee Research Centre, Agroscope Liebefeld-Posieux ALP, Schwarzenburgstrasse 161, 3003 Bern, Switzerland

${ }^{5}$ E-mail: christoph.sandrock@alp.admin.ch

${ }^{6}$ Swiss Federal Institute of Aquatic Science and Technology (EAWAG),

Überlandstrasse 133, Postfach 611, 8600 Dübendorf, Switzerland

${ }^{7}$ E-mail: christoph.vorburger@eawag.ch

*Corresponding author 


\begin{abstract}
Because of the twofold cost of sex, genes conferring asexual reproduction are expected to spread rapidly in sexual populations. However, in reality this simple prediction is often confounded by several complications observed in natural systems. Motivated by recent findings in the Cape honeybee and in the parasitoid wasp Lysiphlebus fabarum, we explore through mathematical models the spread of a recessive, parthenogenesis inducing allele in a haplodiploid population. The focus of these models is on the intricate interactions between the mode of parthenogenesis induction through automixis and complementary sex determination (CSD) systems. These interactions may result in asexual production of diploid male offspring and the spread of the parthenogenesis inducing allele through these males. We demonstrate that if parthenogenetic females produce a substantial proportion of male offspring, this may prevent the parthenogenesis inducing allele from spreading. However, this effect is weakened if these diploid males are at least partially fertile. We also predict a degradation of multilocus CSD systems during the spread of parthenogenesis, following which only a single polymorphic CSD locus is maintained. Finally, based on empirical parameter estimates from L. fabarum we predict that male production in parthenogens is unlikely to prevent the eventual loss of sexual reproduction in this system.
\end{abstract}

Key words: Cape honeybee, central fusion, complementary sex determination, evolution of sex, haplodiploidy, Lysiphlebus fabarum Running title: Contagious parthenogenesis in haplodiploids 


\section{Introduction}

Species or species groups in which closely related sexual and asexual taxa coexist have been regarded as a window of opportunity to study the maintenance of sex, a long-standing problem in evolutionary biology (Maynard Smith 1978; Bell 1982). However, such comparisons are frequently confounded because parthenogenesis is associated with additional differences such as polyploidy or hybridisation. For example, all known asexual vertebrates are hybrids (Vrijenhoek et al. 1989).

More valid comparisons are possible when parthenogenetic lineages arise spontaneously by mutation, for which there is evidence from a number of taxa (Simon et al. 2003). If suppression of sexual reproduction is complete, such a mutation results in the immediate formation of an all-female lineage that is closely related yet reproductively isolated from its bisexual ancestors. More frequently, reproductive isolation is incomplete, either because mutation initiates parthenogenetic lineages still capable of producing males (e.g. Blackman 1972; Innes and Hebert 1988; Turgeon and Hebert 1994; Pongratz et al. 1998), or because the mechanism suppressing sexual reproduction fails occasionally. In these cases, parthenogenesis-inducing alleles may spread through males produced by parthenogenetic lineages. By mating with sexual females, such males may convert sexual into parthenogenetic lineages, a process referred to as 'contagious parthenogenesis' (Simon et al. 2003). Inspired by their work on parthenogenetic earthworms, Jaenike and Selander (1979) modelled the spread of a dominant allele inducing parthenogenetic egg development while permitting normal spermatogenesis. They concluded that such an allele would rapidly go to fixation. This model seems applicable to the spread of obligate parthenogenesis in the cyclical parthenogen Daphnia pulex, although the genetic basis of meiosis suppression is more 
complex in this species, consisting of a dominant epistatic interaction of at least four loci (Lynch et al. 2008).

Mutations inducing parthenogenetic reproduction may not spread as readily if they act recessively. Such a case was described for the Cape honeybee (Apis mellifera capensis), in which parthenogenesis in egg-laying workers appears to be induced by a single recessive allele at the thelytoky locus (Lattorff et al. 2005; Lattorff et al. 2007). We have recently discovered a similar case in the aphid parasitoid Lysiphlebus fabarum (Hymenoptera: Braconidae: Aphidiinae). This wasp is parthenogenetic in most populations (Mackauer and Starý 1967; Starý 1988; Belshaw et al. 1999), but some parthenogenetic lines may spontaneously produce haploid males at very low frequencies. Mating such males to sexual females of the same species and following the reproductive modes of subsequent generations revealed a pattern that was consistent with parthenogenesis being determined by a single recessive allele (C. Sandrock \& C. Vorburger, in prep.).

In both of the above cases, parthenogenetic daughters are produced by a process known as central fusion automixis (Figure 1), where diploidy is restored by fusion of the central products of the meiotic tetrad and metaphase II restitution, respectively (Verma and Ruttner 1983; Belshaw and Quicke 2003). Both automictic processes similarly lead to $50 \%$ homozygous offspring for loci distal to chiasmata if crossing over takes place in the first meiotic division. Other forms of automixis (e.g., terminal fusion, i.e. the fusion of the second polar body nucleus with the egg nucleus) would also produce homozygous offspring to varying degrees (Suomalainen et al. 1987). As a consequence, an interesting clash between parthenogenesis induction and sex determination may ensue in automictic Hymenoptera (Engelstädter 2008). This is 
because Hymenoptera frequently exhibit complementary sex determination (CSD, reviewed in Heimpel and de Boer 2008). In the simplest case, heterozygosity at a single CSD locus determines female development (sl-CSD). sl-CSD occurs for example in honeybees, where the CSD locus has been identified and the sex determination pathways elucidated in detail (Beye et al. 2003; Hasselmann et al. 2008; Gempe et al. 2009). High allelic diversity at the CSD locus ensures that fertilized eggs are generally heterozygous at the CSD locus and develop into diploid females, whereas unfertilized eggs are hemizygous and develop into males. Homozygous diploids, as they may occur under close inbreeding, for example, develop into diploid males. Hence the above-mentioned clash: Automixis also increases homozygosity, which will lead to some proportion of diploid males among parthenogenetically produced offspring if the CSD locus is affected by recombination. This would not be the case under apomictic parthenogenesis.

Diploid males may not survive, as in the honey bee (where they are usually killed: Woyke 1963), they may be sterile (Holloway et al. 1999), but they may also be viable and fertile, as in the vespid wasp Euodynerus foraminatus (Cowan and Stahlhut 2004) or in the parasitoid wasp Cotesia vestalis (de Boer et al. 2007). Such differences are likely to affect the expected rate of spread of a parthenogenesis-inducing allele. The situation is further complicated by the fact that CSD can also include multiple loci (ml-CSD, de Boer et al. 2008), which may reduce or delay diploid male production in automictic parthenogens.

Here, we develop and analyze three models for the spread of a recessive gene coding for parthenogenetic reproduction by automixis in a haplodiploid species with a complementary sex determination system (CSD). The models are of increasing 
complexity and differ in their assumption regarding the CSD system: model 1 assumes a single CSD locus, model 2 assumes multiple, but equivalent CSD loci, and model 3 assumes two CSD loci that may recombine at different rates with the centromere. We also estimate relevant parameters in L. fabarum and based on these estimates and our theoretical results we predict that the parthenogenesis inducing gene is likely to spread in this species.

\section{Models for the spread of parthenogenesis}

\section{Model 1: Single-locus CSD}

We assume a panmictic population with discrete and non-overlapping generations. We model the dynamics of a recessive allele $a$ that induces parthenogenesis in female carriers. The ancestral allele $A$ at the same locus is assumed to have no effect. We denote the frequencies of sexual females with genotypes $A A$ and $A a$ among all females by $p_{A A}$ and $p_{A a}$, respectively. The frequency of parthenogenetic females (with genotype $a a$ ) is denoted by $u$. Finally, there are three types of males possible in the system: haploid males with genotypes $A$ or $a$, and diploid males with genotype $a a$. The frequencies of these males among all males are denoted by $q_{A}, q_{a}$ and $q_{a a}$.

We consider a single-locus CSD system (diploid individuals that are heterozygous at this locus develop into females, whereas haploid individuals or diploid individuals that are homozygous at the CSD locus develop into males). The genetic diversity at the CSD locus is assumed to be sufficiently high in the population so that all fertilized eggs produced by sexual females develop into females. However - depending on the mechanism of parthenogenesis - asexual females may produce offspring that have become homozygous at the CSD locus. The fraction of offspring from an asexual 
female in which this happens is denoted by $\gamma$. The relationship between $\gamma$ and the recombination rate between CSD locus and centromere is explored in Appendix A.

Owing to developmental problems related to asexual reproduction, parthenogenetic females may have a reduced fecundity of $1-s_{f}$ compared to sexually reproducing females. Likewise, diploid ( $a a)$ males have a fertility of $1-s_{m}$ relative to haploid ( $A$ or a) males. We assume that to the extent that diploid males are fertile, they produce haploid sperm only, i.e., we ignore the possibility of triploid offspring arising in our model. Finally, we assume that the primary sex-ratio of sexually produced offspring is 1:1 (resulting in a twofold advantage of asexual reproduction).

Exploiting $p_{A A}=1-p_{A a}-u$ and $q_{A}=1-q_{a}-q_{a a}$, the recursion equations of the dynamical system can be written as

$$
\begin{aligned}
& p_{A a}^{\prime}=\frac{2\left(1-p_{A a}-u\right)\left[q_{a}+\left(1-s_{m}\right) q_{a a}\right]+p_{A a}\left(1-s_{m} q_{a a}\right)}{2(1-u)\left(1-s_{m} q_{a a}\right)+4\left(1-s_{f}\right)(1-\gamma) u}, \\
& u^{\prime}=\frac{p_{A a}\left[q_{a}+\left(1-s_{m}\right) q_{a a}\right]+4\left(1-s_{f}\right)(1-\gamma) u}{2(1-u)\left(1-s_{m} q_{a a}\right)+4\left(1-s_{f}\right)(1-\gamma) u} \\
& q_{a}^{\prime}=\frac{p_{A a}}{2(1-u)+4\left(1-s_{f}\right) \gamma u} \\
& q_{a a}^{\prime}=\frac{4\left(1-s_{f}\right) \gamma u}{2(1-u)+4\left(1-s_{f}\right) \gamma u} .
\end{aligned}
$$

In order to ascertain whether the allele $a$ can invade a population fixed for $A$, we performed a stability analysis of the equilibrium $E_{1}=\left(\hat{p}_{A a}, \hat{u}, \hat{q}_{a}, \hat{q}_{a a}\right)=(0,0,0,0)$. The eigenvalues of the Jacobian matrix evaluated at this fixed point are 


$$
\lambda_{1}=0, \lambda_{2}=1, \lambda_{3}=-1 / 2 \text { and } \lambda_{4}=2(1-\gamma)\left(1-s_{f}\right)
$$

A sufficient condition for an equilibrium to be unstable is that the absolute value of the corresponding leading eigenvalue be greater than one. Thus, if

$$
\lambda_{4}=2(1-\gamma)\left(1-s_{f}\right)>1
$$

$a$ will spread in the population. This is a rather intuitive prediction, as $\lambda_{4}$ is simply the number of daughters that an asexual female produces relative to a sexual female. If $\lambda_{4} \leq 1$, the leading eigenvalue is one. In this case, linear approximation of the dynamics around the equilibrium $E_{1}$ fails to be informative about its stability. However, we were able to derive the following general condition for the spread of $a$ by using higher order terms (see Appendix B):

$$
\left(1-s_{f}\right)\left[2(1-\gamma)+\left(1-s_{m}\right) \gamma\right]>1
$$

This inequality reveals that selection for $a$ can be partitioned into a component arising through the asexual production of daughters (this component is given by $\left.2(1-\gamma)\left(1-s_{f}\right)\right)$ and a second contribution arising through the contagious spread of $a$ through diploid males $\left(\left(1-s_{f}\right)\left(1-s_{m}\right) \gamma\right)$. When $s_{m}=1$ (males are sterile), the contagious component is absent and condition (4) becomes identical to condition (3). Figure 2 illustrates and compares these conditions in terms of the maximum proportion of sons that asexual females may produce if $a$ is to spread in the population.

We next analyzed the stability of the equilibrium $E_{2}=(0,1,0,1)$, corresponding to fixation of $a$. The leading eigenvalue of the Jacobian matrix evaluated at this fixed points is 


$$
\Lambda=\frac{1-s_{m}}{4(1-\gamma)\left(1-s_{f}\right)}
$$

This implies that whenever

$$
\gamma<1-\frac{\left(1-s_{m}\right)}{4\left(1-s_{f}\right)}
$$

$E_{2}$ is stable. Combining this condition with the condition for the $a$ allele to spread in a population reveals that there are four qualitatively different regions of the parameter space in which $a$ spreads or does not spread when rare, and increases or declines in frequency when common (Figure 3A). Interestingly, when $\gamma$ is high but $s_{m}$ is low, $a$ may invade the population and reach a polymorphic equilibrium $E_{3}$. (Note that as shown in Appendix A, the range of values that $\gamma$ can take depends on the mechanism of automixis; for example, with central fusion, $\gamma \leq 1 / 3$, so that no stable polymorphic equilibrium exists.) In the special case of $s_{f}=0$ we were able to obtain an analytical expression for this polymorphic equilibrium, but the formulae are cumbersome and uninformative, and therefore not given here. Extensive numerical screening of the parameter space indicates that no further equilibria in addition to $E_{1}$, $E_{2}$ and $E_{3}$ exist.

The results of the stability analysis of the single CSD locus case can be summarized and interpreted as follows. First, the fecundity of parthenogenetic females must be greater than one half that of sexual females (i.e., $\left.s_{f}<1 / 2\right)$ for $a$ to spread, because otherwise the fecundity cost to parthenogens is greater than the twofold cost of sex. Second, the maximum proportion $\gamma$ of sons that asexual females can produce so that $a$ spreads in the population depends on the relative fertility of $a a$ males. If these males 
are completely sterile (as is observed in some haplodiploid species, see the Discussion), such males may be produced only to the extent that asexual females still produce more daughters than sexual females (Eq. 3). If, however, diploid males can reproduce $\left(s_{m}<1\right)$, the $a$ allele may spread in the population even if condition (3) is not fulfilled, as long as (4) holds. (see Fig. 2). This is because now males are no longer a dead end for $a$; rather, $a a$ males contribute in generating new lines of asexual a $a$ females. (This fitness contribution is quantified by the difference between expressions (3) and (4)). Finally, if $a$ spreads in the population, it may either go to fixation or - in a smaller subset of the parameter space - a stable polymorphism of $a$ and $A$ can be reached so that some females reproduce sexually and others asexually. The reason for the existence of such a stable polymorphism is negative frequency dependent selection for $a$ : when asexual females produce many fertile males (high $\gamma$ and low $s_{\mathrm{m}}$ ), the population sex ratio will become increasingly male biased with increasing frequency of $a$. In line with basic sex ratio theory (Düsing 1883; Fisher 1930), the fitness of $a a$ males will therefore decline with increasing frequency of asexual females in the population relative to the fitness of sexual females, eventually resulting in annihilation of the fitness advantage conferred by $a$.

\section{Model 2: Multi-locus CSD}

We next consider a model with an arbitrary number $M$ of CSD loci. Diploid individuals that are heterozygous at at least one of these loci develop into females, whereas haploid individuals or individuals that are homozygous at all loci develop into males. Again, we make the simplifying assumption that the genetic diversity at the CSD loci is sufficiently high so that all fertilized eggs produced by sexual females are heterozygous at all loci and therefore develop into females. On the other hand, 
asexual females may produce offspring with a reduced level of heterozygosity (from $M$ to zero heterozygous loci). Thus, we partition the class of asexual females in the population into $M$ subclasses according to the number of heterozygous loci. The frequencies of females in these subclasses are denoted by $u_{i}$, where $i$ is the number of heterozygous CSD loci and ranges from 1 to $M$. The fraction of offspring from an asexual female in which a particular CSD locus becomes homozygous is denoted by $\gamma$. This fraction is assumed to be the same for and independent across all loci, leading to a binomial distribution in the number of heterozygous loci among offspring. (Rates of becoming homozygous will be independent if the different loci are located on different linkage groups, or on different arms of the same chromosome.) These assumptions lead to the following recursion equations for the genotypes in the population:

$$
\begin{aligned}
& \bar{W}_{f} p_{A A}^{\prime}=\left(2 p_{A A}+p_{A a}\right) q_{A}, \\
& \bar{W}_{f} p_{A a}^{\prime}=2 p_{A A}\left[q_{a}+\left(1-s_{m}\right) q_{a a}\right]+p_{A a}\left(1-s_{m} q_{a a}\right), \\
& \bar{W}_{f} u_{i}^{\prime}=4\left(1-s_{f}\right) \sum_{k=i}^{M}\left(\begin{array}{c}
k \\
k-i
\end{array}\right) \gamma^{k-i}(1-\gamma)^{i} u_{k} \quad \text { for } i \in\{1,2, \ldots, M-1\}, \\
& \bar{W}_{f} u_{M}^{\prime}=p_{A a}\left[q_{a}+\left(1-s_{m}\right) q_{a a}\right]+4\left(1-s_{f}\right) u_{M}(1-\gamma)^{M}, \\
& \bar{W}_{m} q_{A}^{\prime}=2 p_{A A}+p_{A a}, \\
& \bar{W}_{m} q_{a}^{\prime}=p_{A a}, \\
& \bar{W}_{m} q_{a a}^{\prime}=4\left(1-s_{f}\right) \sum_{k=1}^{M} \gamma^{k} u_{k},
\end{aligned}
$$

where the normalization constants 


$$
\begin{aligned}
& \bar{W}_{f}=2\left(p_{A A}+p_{A a}\right)\left(1-s_{m} q_{a a}\right)+4\left(1-s_{f}\right) \sum_{k=1}^{M}\left(1-\gamma^{k}\right) u_{k} \quad \text { and } \\
& \bar{W}_{m}=2\left(p_{A A}+p_{A a}\right)+4\left(1-s_{f}\right) \sum_{k=1}^{M} \gamma^{k} u_{k}
\end{aligned}
$$

are the mean fitness of females and males, respectively.

Conditions for invasion of the parthenogenesis inducing $a$ allele into a population could only be determined numerically. Figure 3B shows that with increasing number of CSD loci, invasion becomes possible in an increasingly larger proportion of the parameter space. Simulations also show that spread of the $a$ allele becomes faster with increasing number of CSD loci (results not shown).

As in the previous model, we can also ask what the conditions for fixation of $a$ are following invasion. The eigenvalues of the Jacobian matrix, evaluated at the fixed point $E_{2}=\left(\hat{p}_{A A}, \hat{p}_{A a}, \hat{u}_{1}, \hat{u}_{2}, \ldots, \hat{u}_{M}, \hat{q}_{A}, \hat{q}_{a}, \hat{q}_{a a}\right)=(0,0,1,0, \ldots, 0,0,0,1)$ are given by

$$
\Lambda_{i}=(1-\gamma)^{i} \text { for } i=1, \ldots, M-1 \text { and } \Lambda_{M}=\frac{\left(1-s_{m}\right)}{4(1-\gamma)\left(1-s_{f}\right)}
$$

Since $\Lambda_{i}=(1-\gamma)^{i}<1$ for $i=1, \ldots, M-1$ and $\gamma>0$, fixation of $a$ represents a stable fixed point if and only if

$$
\gamma<1-\frac{\left(1-s_{m}\right)}{4\left(1-s_{f}\right)}
$$

which is the same condition as Eq. (6) in the single CSD locus model. Thus, although with increasing number of CSD loci spread of $a$ occurs faster and with larger homozygosity acquisition rates $\gamma$, the conditions for stable fixation of $a$ following its spread are not affected by the number of CSD loci. 
It can further be shown (see Appendix C) that provided the $a$ allele spreads to fixation in the population, heterozygosity in all but one CSD loci will become lost in $a a$ females. (More precisely, the class of a females with a single heterozygous CSD locus will become fixed; this class may in reality be also heterogeneous with respect to which of the loci remains heterozygous.) Figure 4A shows the resulting dynamics of gradual loss of heterozygosity.

\section{Model 3: Two-locus CSD}

We will now assume that there are only two CSD loci, but these loci may be characterized by different rates $\gamma_{1}$ and $\gamma_{2}$ with which these loci may become homozygous, reflecting different proximity of these to their respective centromere and/or different recombination rates on the chromosomes they are located on. As in the previous model, we assume that homozygosity during automixis is acquired independently at the two loci. Denoting by $u_{++}, u_{+-}$and $u_{-+}$the frequencies of parthenogenetic females in which both, only the first or only the second CSD locus are heterozygous, respectively, we arrive at the following recursion equations:

$$
\begin{aligned}
& \bar{W}_{f} p_{A A}^{\prime}=\left(2 p_{A A}+p_{A a}\right) q_{A}, \\
& \bar{W}_{f} p_{A a}^{\prime}=2 p_{A A}\left[q_{a}+\left(1-s_{m}\right) q_{a a}\right]+p_{A a}\left(1-s_{m} q_{a a}\right), \\
& \bar{W}_{f} u_{++}^{\prime}=p_{A a}\left[q_{a}+\left(1-s_{m}\right) q_{a a}\right]+4\left(1-s_{f}\right) u_{++}\left(1-\gamma_{1}\right)\left(1-\gamma_{2}\right), \\
& \bar{W}_{f} u_{+-}^{\prime}=4\left(1-s_{f}\right)\left[u_{+-}\left(1-\gamma_{1}\right)+u_{++}\left(1-\gamma_{1}\right) \gamma_{2}\right] \\
& \bar{W}_{f} u_{-+}^{\prime}=4\left(1-s_{f}\right)\left[u_{-+}\left(1-\gamma_{2}\right)+u_{++} \gamma_{1}\left(1-\gamma_{2}\right)\right] \\
& \bar{W}_{m} q_{A}^{\prime}=2 p_{A A}+p_{A a},
\end{aligned}
$$




$$
\begin{aligned}
& \bar{W}_{m} q_{a}^{\prime}=p_{A a}, \\
& \bar{W}_{m} q_{a a}^{\prime}=4\left(1-s_{f}\right)\left[\gamma_{1} \gamma_{2} u_{++}+\gamma_{1} u_{+-}+\gamma_{2} u_{-+}\right],
\end{aligned}
$$

with

$$
\begin{aligned}
& \bar{W}_{f}=2\left(p_{A A}+p_{A a}\right)\left(1-s_{m} q_{a a}\right)+ \\
& \quad 4\left(1-s_{f}\right)\left[\left(1-\gamma_{1} \gamma_{2}\right) u_{++}+\left(1-\gamma_{1}\right) u_{+-}+\left(1-\gamma_{2}\right) u_{-+}\right], \\
& \bar{W}_{m}=2\left(p_{A A}+p_{A a}\right)+4\left(1-s_{f}\right)\left[\gamma_{1} \gamma_{2} u_{++}+\gamma_{1} u_{+-}+\gamma_{2} u_{-+}\right] .
\end{aligned}
$$

The eigenvalues of the Jacobian matrix at the fixed point

$$
\begin{gathered}
E_{2}=\left(\hat{p}_{A A}, \hat{p}_{A a}, \hat{u}_{++}, \hat{u}_{+-}, \hat{u}_{-+}, \hat{q}_{A}, \hat{q}_{a}, \hat{q}_{a a}\right)=(0,0,0,1,0,0,0,1) \text { can be determined to be } \\
\Lambda_{1}=\frac{\left(1-s_{m}\right)}{4\left(1-\gamma_{1}\right)\left(1-s_{f}\right)} \text { and } \Lambda_{2}=\frac{1-\gamma_{2}}{1-\gamma_{1}} .
\end{gathered}
$$

The first of these eigenvalues is equivalent to $\Lambda$ (eq. 5) and $\Lambda_{M}$ (eq. 9) obtained in the two previous models and demonstrates once more that if $\gamma_{1}$ is very high, a polymorphic equilibrium may become the globally stable fixed point of the system. The second eigenvalue, $\Lambda_{2}$, shows that fixation of the +- genotype is only stable if $\gamma_{1}<\gamma_{2}$. Conversely, and evident by the symmetry of the system, fixation of the -+ genotype is only stable if $\gamma_{2}<\gamma_{1}$. Thus, provided the $a$ allele can invade the population and $\gamma_{1}$ or $\gamma_{2}$ fulfill condition (6), the genotype associated with the lower $\gamma_{i}$ will become fixed in the population. For example, with automixis through central fusion, this result implies that only the CSD locus that is closest to its centromer will remain polymorphic. An example for the dynamics in this system is provided in Figure 4B. 


\section{Parameter estimates for Lysiphlebus fabarum}

The models described above identified $s_{f}, s_{m}$ and $\gamma$ as important parameters determining the spread of a parthenogenesis-inducing allele $a$. Our work to elucidate the genetic basis of parthenogenesis in the aphid parasitoid L. fabarum allowed us to obtain rough estimates of these parameters for that specific study system. The detailed data will be published elsewhere. Briefly, we used experimental crosses to produce females homozygous for a recessive, parthenogenesis-inducing allele (corresponding to $a$ a females in our model). To obtain an estimate of $s_{f}$, the number of offspring produced by these parthenogenetic females was compared with the number of offspring produced by their unmated sexual sisters ( $a A$ or $A A$ females). Host aphids were provided ad libitum. Note that the number of offspring is a very inclusive fitness estimate for a parasitoid female, as it is not only influenced by fecundity, but also by oviposition success and the offsprings' ability to develop in the host. Parthenogenetic females produced $58.45 \pm 3.27$ (SE) offspring on average, their sexual sisters only $48.91 \pm 3.08$ (Sandrock, et al., in prep.). This suggests an increased rather than reduced reproductive success of parthenogenetic females (i.e. $s_{f}<0$ ). However, unmated sexual females could only produce sons and may thus have been less motivated to oviposit than their parthenogenetic sisters. Therefore, we also measured offspring production in mated, sexual females. They produced $59.14 \pm 4.58$ offspring on average, slightly but not significantly more than the thelytokous females $\left(t_{197}=\right.$ $0.125, P=0.901)$. This indicates that the fecundity reduction of thelytokous females in L. fabarum, if present at all, is very small $\left(s_{f} \approx 0.01\right)$.

To obtain an estimate of $s_{m}$, the fertility reduction of diploid compared to haploid males, we compared sex ratios of sexual females that had mated either with a haploid 
or a diploid male. Sexual females produce sons from unfertilized eggs and daughters from fertilized eggs. A lower proportion of daughters in females mated to diploid males would thus be indicative of their reduced fertility. Females mated to haploid males produced $75.6 \pm 1.1 \%$ daughters, those mated to diploid males produced 73.5 $\pm 1.8 \%$ daughters on average. This difference was not statistically significant $\left(t_{78}=\right.$ $0.829, P=0.410)$. Hence, diploid males in L. fabarum seem to suffer only from a small reduction in fertility, if any $\left(s_{m} \approx 0.03\right)$ (Sandrock et al., in prep.).

Finally, we tried to estimate $\gamma$, the homozygosity acquisition rate at which automictic parthenogenetic females produce diploid males. For this we used six different thelytokous lines of L. fabarum (A - F), which were founded by single a a females obtained in the laboratory from experimental crossings. The proportion of males they produced was determined for between 14 and 23 generations. These proportions were $0.349 \pm 0.014$ (line A), $0.323 \pm 0.013(B), 0.188 \pm 0.006(C), 0.167 \pm 0.007(D)$ $0.087 \pm 0.008(\mathrm{E})$, and $0.071 \pm 0.011(\mathrm{~F})$ (Sandrock et al., in prep.). Note that the two highest estimates are not significantly different from $1 / 3$, the expected maximum value under central fusion automixis (see Appendix A) (one-sample t-tests, both $P>$ $0.25)$.

Thus, our empirical estimates of $\gamma$ for $L$. fabarum range from about 0.07 to 0.35 , with a mean of 0.22 . The large differences among these proportions for different isofemale lines are suggestive of multilocus CSD in L. fabarum and might reflect that different lines remained heterozygous at different CSD loci, as predicted by Model 2.

Considering the estimates for all three parameters together and comparing them with the results from our models (see also Figures 2 and 3), it appears that even the highest estimates of $\gamma$ would be insufficient to prevent the spread of a recessive, 
parthenogenesis-inducing allele in L. fabarum. This is because the high fitness of thelytokous females and diploid males observed in this system are favourable for the spread of such an allele.

\section{Discussion}

\section{Automixis \& sex determination}

If parthenogenetic females produce diploid eggs through automixis rather than ameiotically, this has the side effect of increased homozygosity among the offspring (Suomalainen et al. 1987) (see Appendix A for a quantification of this effect for the various forms of automixis). As a consequence, automixis can lead to reduced fitness of offspring due to the unmasking of deleterious recessive mutations, i.e., a special type of inbreeding depression (Archetti 2004; Engelstädter 2008). Although we have not studied this type of inbreeding depression explicitly in our models, it is accounted for to some extent by our parameter $s_{f}$, denoting the fecundity reduction of parthenogenetic females. However, we do not expect strong inbreeding depression in the system we studied because in haplodiploids, recessive deleterious mutations are efficiently purged from the population through exposure in males (Crozier 1985; Werren 1993). The low parameter estimate for $s_{f}$ obtained from L. fabarum is in accord with this expectation.

Another consequence of an increase of homozygosity through automixis is its potential impact on sex determination (Maynard Smith 1978; Engelstädter 2008). Such an impact is expected in species where the female sex is determined by heterozygosity at one or more loci, as occurs in ZW sex determination systems (like in birds and butterflies) and complementary sex determination (CSD) in many 
hymenopterans. As our models demonstrate for the case of CSD, the asexual production of diploid sons through increased homozygosity can impede the spread of an allele that induces parthenogenesis. The magnitude of this effect depends crucially on the fitness of the males produced through automixis. If these males are not viable or infertile, the parthenogenesis-inducing allele may not spread because not enough parthenogenetic daughters are produced. Infertile diploid males are indeed usually observed in the Hymenoptera (Macbride 1946; El Agoze et al. 1994; Holloway et al. 1999; Krieger et al. 1999), and active killing of diploid males by workers occurs in ants, wasps and bees (Woyke 1963, 1979; Pamilo et al. 1994; Henshaw et al. 2002). On the other hand, diploid males can also be viable and highly fertile (e.g., Cowan and Stahlhut 2004). Our results show that if this is the case, parthenogenesis can invade the population even if a substantial proportion of the asexually produced offspring are males. This is because diploid males can now further transmit the parthenogenesis inducing allele and establish new asexual lines (a process that has been termed 'contagious parthenogenesis': Simon et al. 2003). This should be the case in L. fabarum, because diploid males have a high fertility (see above). It will therefore be interesting to study how sexual populations, although they are much less common than asexuals, are maintained in this species.

The sex determination system not only influences the spread of genes inducing automixis, but can also in turn be modified through the spread of automixis in the population. In particular, automixis is expected to have a meltdown effect on multilocus CSD systems in the parthenogenetic females. This is because an increasing number of loci will become homozygous over time, eventually reducing the multilocus CSD system to single-locus CSD. Thus, the proportion of males produced by the parthenogenetic females will increase over time as the CSD system is eroded. Our 
model further predicts that the one locus that remains heterozygous in the population is the one that has the lowest probability of becoming homozygous through automixis (e.g., the one closest to its centromere when automixis is achieved through central fusion and recombination rates are invariant across chromosomes). However, this result may be confounded by stochastic effects during the early phase of the spread of the parthenogenesis-inducing allele, in that asexual lines producing fewer males become extinct through random genetic drift and a line producing comparatively more males eventually becomes fixed in the population.

We expect selection to act on recombination rates in parthenogenetic females (reduced recombination between CSD loci and the centromere in the case of central fusion automixis), so that the proportion of males produced by these females is reduced. Selection for reduced male production might explain why in field-collected parthenogenetic lines of $L$. fabarum, the proportion of male offspring is typically very low ( $\leq 1 \%$, Belshaw et al. 1999; Starý 1999, own unpublished data), whereas the new parthenogenetic lines we generated by experimental crosses produced between close to zero and $35 \%$ males.

\section{Limitations of the models}

In order to keep our models as simple as possible, we made a number of assumptions that may be violated in natural systems. For example, we assumed that all females homozygous for the parthenogenesis-inducing gene reproduce exclusively through automixis. By contrast, some parthenogenetic females of L. fabarum have been observed to produce haploid eggs at a low rate (C. Sandrock and C. Vorburger, in prep.), indicating that the penetrance of automixis induction is not perfect. Clearly, reduced penetrance of parthenogenesis will lead to reduced selection for the 
parthenogenesis-inducing allele. A second assumption of our models is that diploid males produce only diploid daughters, as has been reported for example in the solitary wasp Euodynerus foraminatus (Cowan and Stahlhut 2004) or the braconid parasitoid Cotesia glomerata (Elias et al. 2009). However, in most hymenopterans, diploid males produce diploid sperm, which can give rise to triploid daughters (e.g., de Boer et al. 2007; Cournault and Aron 2009). Given that triploid females are likely to have reduced viability and/or fecundity, we expect that including triploid females into the model should have a similar effect as assuming reduced fertility of diploid males, i.e., the spread of the parthenogenesis inducing allele should be decelerated and the conditions for this spread should become more stringent.

We also assumed a primary sex-ratio of 1:1 for the offspring of sexual individuals. However, in L. fabarum, as in many parasitoid wasps, sex-ratios tend to be female biased (e.g., Hamilton 1967). Such a situation will reduce the cost of sex below twofold, thus slowing down the spread of alleles inducing parthenogenesis. Unfortunately, simply introducing an additional parameter to the models specifying an arbitrary primary sex-ratio would not improve the realism of the models, which is why we have refrained from doing so here. The reason for this is that a female-biased sex-ratio that is not selected for (e.g., through local mate competition) could provide diploid males produced by asexual females an undue advantage in the population. Because of this effect, explicit modeling of the factors that produce a sex-ratio different from 1:1 is necessary to investigate the impact of sex-ratio on the spread of parthenogenesis in haplodiploids, which is beyond the scope of the present study.

In conclusion, our models predict that a recessive parthenogenesis inducing allele will spread in a haplodiploid population unless (1) parthenogenetic females have a 
substantially reduced fecundity, (2) these females produce a large proportion of diploid male offspring through increased homozygosity at CSD loci, and (3) these diploid males have strongly reduced fertility. With multi-locus CSD as the underlying sex determination system, we expect a meltdown of this CSD system towards singlelocus CSD in asexual lines, i.e., only a single CSD locus will remain polymorphic.

\section{Acknowledgements}

We thank Alexandre Gouskov for his assistance in determining parasitoid offspring numbers and sex ratios, Gabriel Leventhal for discussion concerning the stability analysis, and two anonymous reviewers for helpful comments on the manuscript. This work was supported by grants from the Swiss National Science Foundation to C.V. 


\section{Appendix A: The relationship between recombination rate and the rate of becoming homozygous}

Let us denote by $r(n)$ the proportion of offspring in which recombination between a given CSD locus and the centromere occurs, depending on the number $n$ of crossover events. We then have

$$
r(0)=0 \quad \text { and } \quad r(n)=\sum_{i=1}^{n}\left(-\frac{1}{2}\right)^{i-1}=\frac{2}{3}\left(1-\left(-\frac{1}{2}\right)^{n}\right) \text { for } n \geq 1
$$

If we now assume that the number of crossover events follows a Poisson distribution with mean $\tilde{n}$, we get as the mean proportion of recombinants

$$
\tilde{r}(\tilde{n})=\sum_{k=0}^{\infty} \frac{\tilde{n}^{k} e^{-\tilde{n}}}{k !} r(k)=\frac{2}{3} \sum_{k=1}^{\infty} \frac{\tilde{n}^{k} e^{-\tilde{n}}}{k !}\left(1-\left(-\frac{1}{2}\right)^{k}\right)=\frac{2}{3}\left(1-e^{-3 \tilde{n} / 2}\right) .
$$

Thus, with increasing number of crossovers, the proportion of recombinants converges to $2 / 3$. This result can also be understood intuitively as follows (see also Figure 1 and Oldroyd et al. 2008). Consider a heterozygous locus and a focal gene copy at this locus on one of the four chromatids. There are three other gene copies that the focal gene copy can become associated with following the first meiotic division. Of these three gene copies, one will be identical to the focal gene copy, and two will be different. Given that with free recombination between centromere and locus (i.e., large $n$ ) the probability of being picked is the same for all of these three gene copies, the probability of obtaining a recombinant product of Meiosis I will be $2 / 3$. 
Depending on the mechanism of automixis, the proportion $\gamma$ of offspring in which a formerly heterozygous CSD locus has become homozygous can now be calculated. With gamete duplication (GD), recombination has no effect, and $\gamma_{\mathrm{GD}}=1$. With terminal fusion (TF), homozygotes are produced always and only when there is no recombination, so that

$$
\gamma_{\mathrm{TF}}=1-\tilde{r}(\tilde{n})=\frac{1}{3}\left(1+2 e^{-3 \tilde{n} / 2}\right)
$$

This indicates that at least a fraction of $1 / 3$ homozygotes will be produced with terminal fusion (even with maximum recombination), and with close linkage between CSD locus and centromere, all offspring will be homozygous. With central fusion (CF) $50 \%$ of the offspring will be homozygous if recombination occurred during meiosis, but none otherwise, yielding

$$
\gamma_{\mathrm{CF}}=\tilde{r}(\tilde{n}) / 2=\frac{1}{3}\left(1-e^{-3 \tilde{n} / 2}\right)
$$

Here, no homozygotes are produced when linkage between CSD locus and centromere is tight ( $\operatorname{small} \tilde{n}$ ), but as many as $1 / 3$ of the offspring will be homozygous if recombination rate is high.

Finally, with random fusion of meiotic products, 1/3 of offspring will be homozygous regardless of the recombination rate. 


\section{Appendix B: Stability of the equilibrium $E_{1}$ in model 1}

Here, we derive the condition for the equilibrium $E_{1}=\left(\hat{p}_{A a}, \hat{u}, \hat{q}_{a}, \hat{q}_{a a}\right)=(0,0,0,0)$ in model 1 to be unstable, i.e., the condition for the $a$ allele to spread when rare. Starting from the recursion equations (1), we first replace the mean fitness in females and males (denominators in the equations) by 2 , which is a good approximation when $p_{A a}, u, q_{a}$ and $q_{a a}$ are all small. We further simplify the system by ignoring all quadratic terms that involve either $u$ or $q_{a a}$; this is justified by the observation that when $p_{A a}$ and $q_{a}$ are initially of order $\varepsilon, u$ and $q_{a a}$ will be of order $\varepsilon^{2}$. These assumptions lead to the following simplified, but still non-linear system of recursion equations that approximate the dynamics for small frequencies of the allele $a$ :

$$
\begin{aligned}
& p_{A a}^{\prime} \approx \frac{p_{A a}}{2}+q_{a}+\left(1-s_{m}\right) q_{a a}-p_{A a} q_{a}, \\
& u^{\prime} \approx 2\left(1-s_{f}\right)(1-\gamma) u+\frac{p_{A a} q_{a}}{2}, \\
& q_{a}^{\prime} \approx p_{A a} / 2, \\
& q_{a a}^{\prime} \approx 2\left(1-s_{f}\right) \gamma u .
\end{aligned}
$$

In a second step, we exploit the fact that $q_{a} \approx p_{A a} / 2$ and $q_{a a} \approx 2\left(1-s_{f}\right) \gamma u$ when changes in genotype frequencies from one generation to the next are small. Inserting these expressions into the recursion equations for the genotype frequencies in females reduces the dimension of the system to two, yielding

$$
p_{A a}^{\prime} \approx p_{A a}\left(1-\frac{p_{A a}}{2}\right)+2 \gamma\left(1-s_{f}\right)\left(1-s_{m}\right) u
$$




$$
u^{\prime} \approx 2\left(1-s_{f}\right)(1-\gamma) u+\frac{p_{A a}^{2}}{4}
$$

These expressions show that with a frequency of the $a$ allele in females of $p_{A a} / 2+u$, the frequency of $a$ in the next generation will be

$$
p_{A a}^{\prime} / 2+u^{\prime} \approx p_{A a} / 2+\left(1-s_{f}\right)\left[2(1-\gamma)+\gamma\left(1-s_{m}\right)\right] u \text {. }
$$

Thus, for $a$ to increase in frequency from one generation to the next,

$$
\left(1-s_{f}\right)\left[2(1-\gamma)+\gamma\left(1-s_{m}\right)\right]>1
$$

must hold.

To verify condition (B4), we performed a numerical screening of the parameter space in the following manner. First, we randomly chose 500 combinations of the parameters $s_{f}$ (from a uniform distribution with range $\left.[0,0.5]\right)$ and $s_{m}$ (from $[0,1]$ ). For each of these parameter combinations, we then calculated the critical value of $\gamma$ by setting the left side of inequality (B4) to 1, yielding $\gamma_{\text {crit }}=\left(1-2 s_{f}\right) /\left[\left(1-s_{f}\right)\left(1+s_{m}\right)\right]$. We then iterated the recursion equations (1) for each parameter combination of $s_{f}$ and $s_{m}$ and with either $\gamma=0.99 \gamma_{\text {crit }}$ (in which case the $a$ allele should spread in the population) or $\gamma=1.01 \gamma_{\text {crit }}$ (in which case $a$ is not expected to spread). Initial genotype frequencies were $\left(p_{A a}, q_{a}, u, q_{a a}\right)=\left(10^{-6}, 0,0.5 \times 10^{-6}, 0\right)$. Following $10^{6}$ generations, we recorded the frequency of the $a$ allele among females and compared this to the initial allele frequency. We found that with $\gamma=0.99 \gamma_{\text {crit }}, a$ always increased in frequency by at least $0.3 \%$, whereas with $\gamma=1.01 \gamma_{\text {crit }}, a$ always decreased in frequency by at least 
$0.3 \%$. This indicates that condition (B4) does indeed predict the spread or non-spread of a rare allele $a$ with high accuracy.

\section{Appendix C: Stability analysis in model 2}

Here, we show that in the multilocus CSD model the only equilibrium upon fixation of the $a$ allele is fixation of the class of genotypes in which all but one CSD locus have become homozygous. When the $a$ allele is fixed in the population (i.e., $\left.p_{A A}=p_{A a}=0\right)$, the recursion equations for the females in the population simplify to

$$
u_{i}^{\prime}=\sum_{k=i}^{M}\left(\begin{array}{c}
k \\
k-i
\end{array}\right) \gamma^{k-i}(1-\gamma)^{i} u_{k} / \sum_{k=1}^{M}\left(1-\gamma^{k}\right) u_{k}
$$

Let us assume now that this dynamical system has a fixed point $\hat{\mathbf{u}}=\left(\hat{u}_{1}, \hat{u}_{2}, \ldots, \hat{u}_{M}\right)$ with $\hat{u}_{M}>0$. We then have

$$
\hat{u}_{M}=(1-\gamma)^{M} \hat{u}_{M} / \sum_{k=1}^{M}\left(1-\gamma^{k}\right) \hat{u}_{k} \quad \Leftrightarrow \quad \sum_{k=1}^{M}\left(1-\gamma^{k}\right) \hat{u}_{k}=(1-\gamma)^{M}
$$

However, since

$$
\sum_{k=1}^{M}\left(1-\gamma^{k}\right) \hat{u}_{k} \geq(1-\gamma)>(1-\gamma)^{M}
$$

eq. (C2) is never fulfilled. Thus, we arrive at a contradiction to the assumption that $\hat{u}_{M}>0$, proving that $\hat{u}_{M}=0$ must hold for any fixed point $\hat{\mathbf{u}}$ of eq. (C1). Reducing the system from $M$ to $M-1$ dimensions and applying the same argument as above demonstrates that the only equilibrium of $(\mathrm{C} 1)$ is $\hat{\mathbf{u}}=(1,0, \ldots, 0)$. 


\section{References}

Archetti, M. 2004. Recombination and loss of complementation: a more than two-fold cost for parthenogenesis. J. Evol. Biol. 17:1084-1097.

Bell, G. 1982. The Masterpiece of Nature: the Evolution and Genetics of Sexuality. University of California Press, Berkeley.

Belshaw, R., and D. L. J. Quicke. 2003. The cytogenetics of thelytoky in a predominantly asexual parasitoid wasp with covert sex. Genome. 46:170-173.

Belshaw, R., D. L. J. Quicke, W. Völkl, and H. C. J. Godfray. 1999. Molecular markers indicate rare sex in a predominantly asexual parasitoid wasp. Evolution 53:1189-1199.

Beye, M., M. Hasselmann, M. K. Fondrk, R. E. Page, and S. W. Omholt. 2003. The gene csd is the primary signal for sexual development in the honeybee and encodes an SR-type protein. Cell 114:419-429.

Blackman, R. L. 1972. The inheritance of life-cycle differences in Myzus persicae (Sulz.) (Hem., Aphididae). Bull. Entomol. Res. 62:281-294.

Cournault, L., and S. Aron. 2009. Diploid males, diploid sperm production, and triploid females in the ant Tapinoma erraticum. Naturwissenschaften 96:13931400.

Cowan, D. P., and J. K. Stahlhut. 2004. Functionally reproductive diploid and haploid males in an inbreeding hymenopteran with complementary sex determination. Proc. Natl. Acad. Sci. USA 101:10374-10379. 
Crozier, R. H. 1985. Adaptive consequences of male-haploidy. Pp. 201-222 in W. Helle, and M. W. Sabelis, eds. Spider Mites: Their Biology, Natural Enemies and Control. Elsevier, Amsterdam.

de Boer, J. G., P. J. Ode, A. K. Rendahl, L. E. M. Vet, J. B. Whitfield, and G. E. Heimpel. 2008. Experimental support for multiple-locus complementary sex determination in the parasitoid Cotesia vestalis. Genetics. 180:1525-1535.

de Boer, J. G., P. J. Ode, L. E. M. Vet, J. B. Whitfield, and G. E. Heimpel. 2007. Diploid males sire triploid daughters and sons in the parasitoid wasp Cotesia vestalis. Heredity 99:288-294.

Düsing, K. 1883. Die Factoren, welche die Sexualität entscheiden. Jenaische Zeitschrift für Naturwissenschaft 16 428-464.

El Agoze, M., J. M. Drezen, S. Renault, and G. Periquet. 1994. Analysis of the reproductive potential of diploid males in the wasp Diadromus pulchellus (Hymenoptera, Ichneumonidae). Bull. Entomol. Res. 84:213-218.

Elias, J., D. Mazzi, and S. Dorn. 2009. No Need to Discriminate? Reproductive Diploid Males in a Parasitoid with Complementary Sex Determination. Plos One 4.

Engelstädter, J. 2008. Constraints on the evolution of asexual reproduction. Bioessays 30:1138-1150.

Fisher, R. A. 1930. The Genetical Theory of Natural Selection. Oxford University Press, Oxford. 
Gempe, T., M. Hasselmann, M. Schiott, G. Hause, M. Otte, and M. Beye. 2009. Sex Determination in Honeybees: Two Separate Mechanisms Induce and Maintain the Female Pathway. PLoS Biol. 7.

Hamilton, W. D. 1967. Extraordinary Sex Ratios. Science 156 477-488.

Hasselmann, M., T. Gempe, M. Schiott, C. G. Nunes-Silva, M. Otte, and M. Beye. 2008. Evidence for the evolutionary nascence of a novel sex determination pathway in honeybees. Nature 454:519-U517.

Heimpel, G. E., and J. G. de Boer. 2008. Sex determination in the Hymenoptera. Annu. Rev. Entomol. 53:209-230.

Henshaw, M. T., D. C. Queller, and J. E. Strassmann. 2002. Control of male production in the swarm-founding wasp, Polybioides tabidus. J. Evol. Biol. $15: 262-268$.

Holloway, A. K., G. E. Heimpel, M. R. Strand, and M. F. Antolin. 1999. Survival of diploid males in Bracon sp. near hebetor (Hymenoptera: Braconidae). Ann. Entomol. Soc. Am. 92:110-116.

Innes, D. J., and P. D. N. Hebert. 1988. The origin and genetic basis of obligate parthenogenesis in Daphnia pulex. Evolution. 42:1024-1035.

Jaenike, J., and R. K. Selander. 1979. Evolution and ecology of parthenogenesis in earthworms. Am. Zool. 19:729-737.

Krieger, M. J. B., K. G. Ross, C. W. Y. Chang, and L. Keller. 1999. Frequency and origin of triploidy in the fire ant Solenopsis invicta. Heredity 82:142-150. 
Lattorff, H. M. G., R. F. A. Moritz, R. M. Crewe, and M. Solignac. 2007. Control of reproductive dominance by the thelytoky gene in honeybees. Biol. Lett. 3:292295.

Lattorff, H. M. G., R. F. A. Moritz, and S. Fuchs. 2005. A single locus determines thelytokous parthenogenesis of laying honeybee workers (Apis mellifera capensis). Heredity. 94:533-537.

Lynch, M., A. Seyfert, B. Eads, and E. Williams. 2008. Localization of the genetic determinants of meiosis suppression in Daphnia pulex. Genetics. 180:317-327.

Macbride, D. H. 1946. Failure of sperm of Habrobracon diploid males to penetrate the eggs. Genetics 31:224-224.

Mackauer, M., and P. Starý. 1967. World Aphidiidae. Le François, Paris.

Maynard Smith, J. 1978. The Evolution of Sex. Cambridge University Press, Cambridge.

Oldroyd, B. P., M. H. Allsopp, R. S. Gloag, J. Lim, L. A. Jordan, and M. Beekman. 2008. Thelytokous parthenogenesis in unmated queen honeybees (Apis mellifera capensis): Central fusion and high recombination rates. Genetics 180:359-366.

Pamilo, P., L. Sundstrom, W. Fortelius, and R. Rosengren. 1994. Diploid males and colony-level selection in Formica ants. Ethol. Ecol. Evol. 6:221-235.

Pongratz, N., T. F. Sharbel, L. W. Beukeboom, and N. K. Michiels. 1998. Allozyme variability in sexual and parthenogenetic freshwater planarians: evidence for 
polyphyletic origin of parthenogenetic lineages through hybrdidization with coexisting sexuals. Heredity. 81:37-47.

Simon, J. C., F. Delmotte, C. Rispe, and T. Crease. 2003. Phylogenetic relationships between parthenogens and their sexual relatives: the possible routes to parthenogenesis in animals. Biol. J. Linn. Soc. 79:151-163.

Starý, P. 1988. Aphidiidae. Pp. 171-184 in A. K. Minks, and P. Harrewijn, eds. Aphids: Their Biology, Natural Enemies, and Control. Vol. 2B. Elsevier, Amsterdam.

Starý, P. 1999. Biology and distribution of microbe-associated thelytokous populations of aphid parasitoids (Hym., Braconidae, Aphidiinae). Journal of Applied Entomology-Zeitschrift Fur Angewandte Entomologie 123:231-235.

Stenberg, P., and A. Saura. 2009. Cytology of Asexual Animals. Pp. 63-74 in I. Schön, K. Martens, and P. van Dijk, eds. Lost Sex - The Evolutionary Biology of Parthenogenesis. Springer, Dordrecht.

Suomalainen, E., A. Saura, and J. Lokki. 1987. Cytology and Evolution in Parthenogenesis. CRC Press, Boca Raton, Florida.

Turgeon, J., and P. D. N. Hebert. 1994. Evolutionary interactions between sexual and all-female taxa of Cyprinotus (Ostracoda: Cyprididae). Evolution. 48:18551865 .

Verma, S., and F. Ruttner. 1983. Cytological analysis of the thelytokous parthenogenesis in the Cape honeybee (Apis mellifera capensis Escholtz). Apidologie. 14:41-57. 
Vrijenhoek, R. C., R. M. Dawley, C. J. Cole, and J. P. Bogart. 1989. A list of known unisexual vertebrates. Pp. 19-23 in R. M. Dawley, and J. P. Bogart, eds. Evolution and Ecology of Unisexual Vertebrates. New York State Museum, Albany, New York.

Werren, J. H. 1993. The evolution of inbreeding in haplodiploid organisms. Pp. 42-73 in N. W. Thornhill, ed. The Natural History of Inbreeding and Outbreeding. The University of Chicago Press, Chicago.

Woyke, J. 1963. What happens to diploid drone larvae in a honeybee colony? J. Apic. Res. 2:73-75.

Woyke, J. 1979. Sex determination in Apis cerana india. J. Apic. Res. 18:122-127. 
Figure 1: Illustration of central fusion automixis, (A) without and (B) with recombination. (A) A pair of chromosomes carrying a heterozygous locus (alleles shown as empty and filled squares) is considered. Following meiosis, two central products of the meiotic tetrad fuse to form a diploid cell. Heterozygosity is maintained in this case. (B) A crossover between the focal locus and the centromere takes place during Prophase I. As a result, fusion of two central meiotic products may - with probability $1 / 2-$ lead to homozygosity. Consequently, if the focal locus is the sex determination locus in a slCSD system, the zygote in (B) will develop into a male. For a more detailed description of central fusion and other mechanisms of automictic parthenogenesis, see Suomalainen et al. (1987) and Stenberg \& Saura (2009).

Figure 2: Maximum homozygosity acquisition rate $\gamma$ in the single CSD locus model that permits spread of the parthenogenesis-inducing allele $a$. The maximum value of $\gamma$ is given as a function of $s_{\mathrm{f}}$ and $s_{\mathrm{m}}$ by the upper, blue surface, derived from inequality (4). For comparison, the lower, yellow surface gives the prediction based on inequality (3), which constitutes a sufficient, but not necessary condition for spread of $a$ that only takes the asexual production of daughters, but not the contagious spread of $a$ through males into account.

Figure 3: Subdivision of the parameter space according to dynamic behaviour. (A) Single CSD locus model. Below the solid line, $a$ spreads in the population when rare, whereas above the solid line, $a$ is selected against when rare. Below the dashed line, $a$ increases in frequency when near fixation, whilst above the dashed line, the frequency of $a$ declines when common. These stability conditions result in four qualitatively different subsets of the parameter space exist, as indicated by diagrams of the dynamics of the parthenogenesis inducing allele $a$. In these diagrams, empty circles 
denote unstable fixed points, filled circles stable fixed points, and arrows the direction in which the frequency of $a$ changes over time. (B) Model with multiple CSD loci. With increasing number of CSD loci (from one to ten), the curve delimiting whether spread of $a$ is possible or not shifts towards greater values of $\gamma$ (solid lines). The curve delimiting stability of fixation of $a$ (dashed line) is not affected by the number of CSD loci. In both plots, $s_{f}=0$. With increasing $s_{f}$, both regions in which invasion of $a$ occurs become smaller (not shown).

Figure 4: Examples for the dynamics of invasion of the parthenogenesis inducing allele $a$ with multi-locus CSD systems. (A) Dynamics in a five-locus system (model 2) showing the frequencies $u_{1}, u_{2}, \ldots, u_{5}$ of $a a$ females with different numbers of heterozygous sex determination loci (colored lines) as well as the total proportion $u_{\text {total }}=\sum_{i=1}^{5} u_{i}$ of asexual females in the population (thick black line). (B) Example for the dynamics in the model with two CSD loci (model 3), showing the frequencies $u_{++}$, $u_{+-}$, and $u_{-+}$of $a a$ females heterozygous at both or either CSD locus (red, blue and green curve, respectively) as well as the total proportion $u_{\text {total }}=u_{++}+u_{+-}+u_{-+}$of asexual females in the population (thick black line). Parameters take the values $s_{f}=0.1, s_{m}=0.5$, and (A) $\gamma=0.02$ or (B) $\gamma_{1}=0.02, \gamma_{2}=0.05$. 


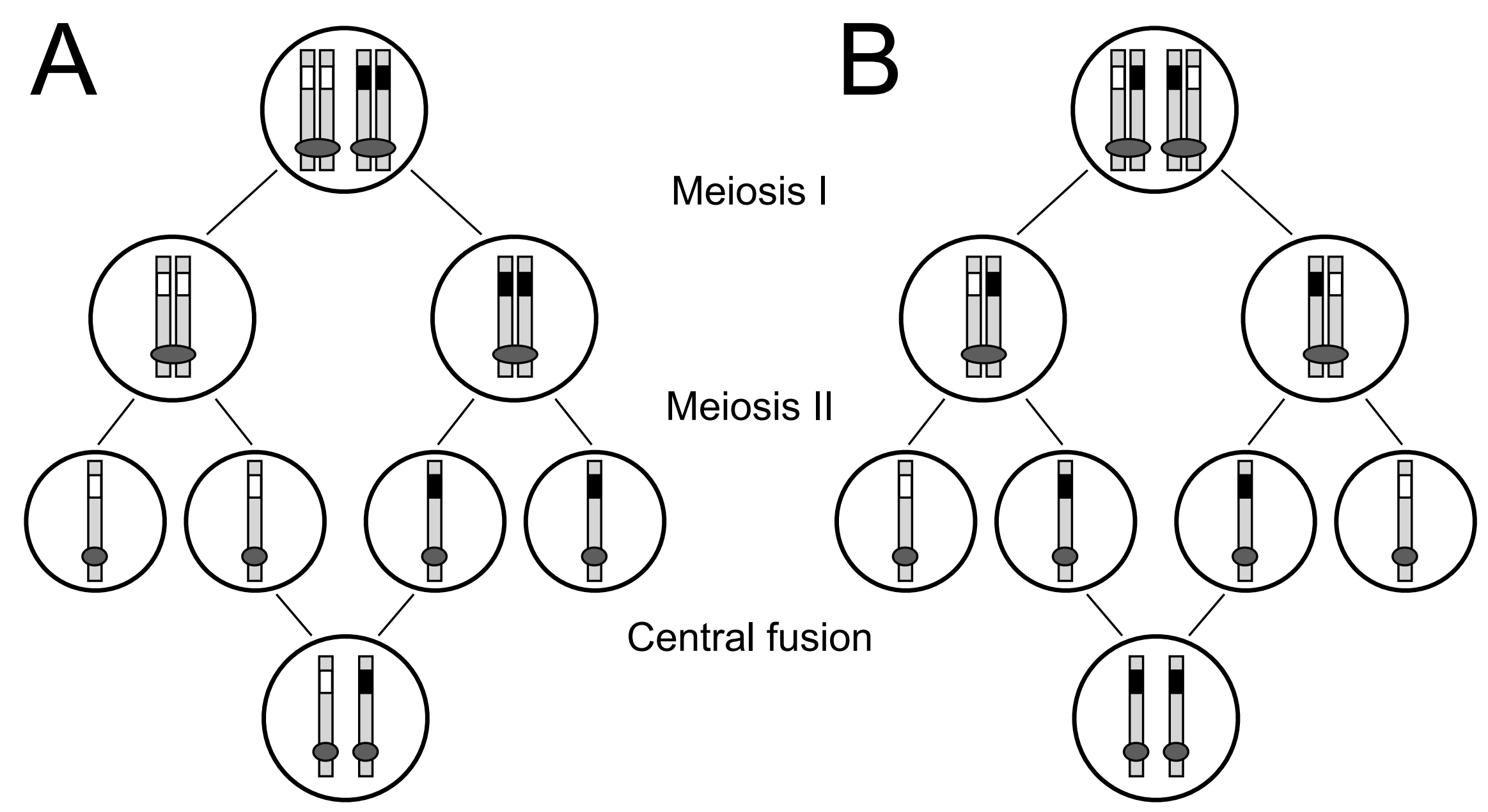




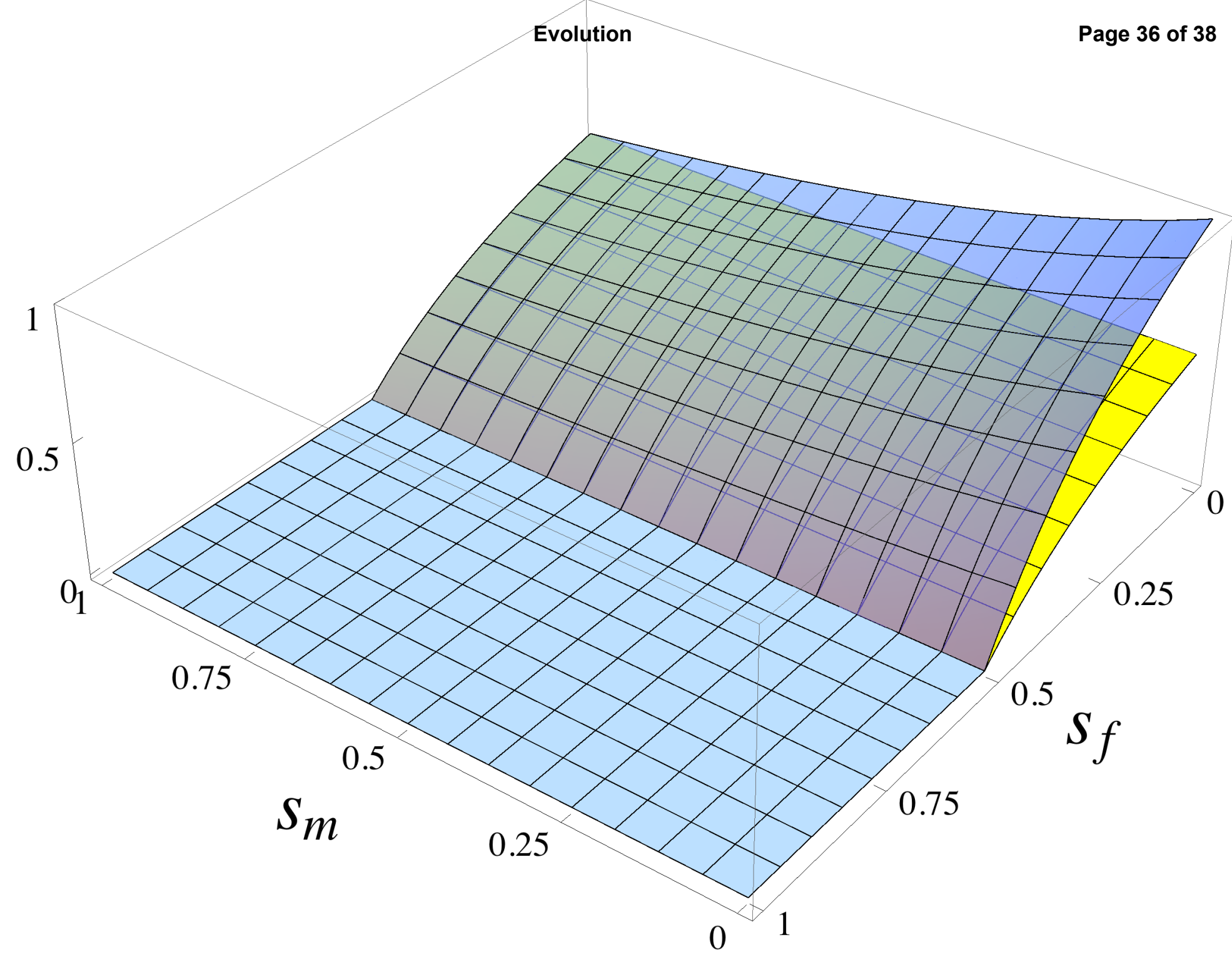




\section{A 37 of $38 \quad$ Evolution}

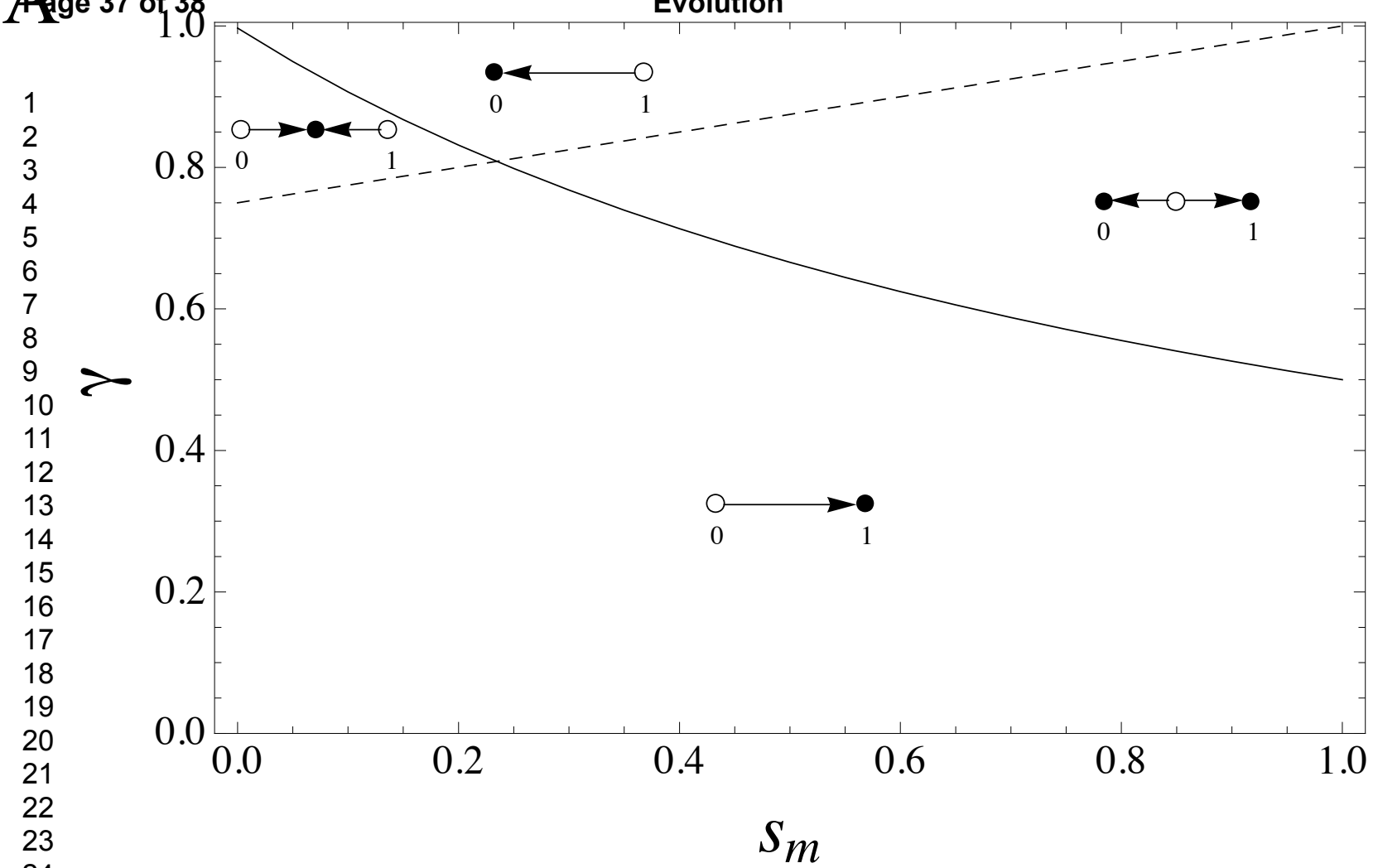

24

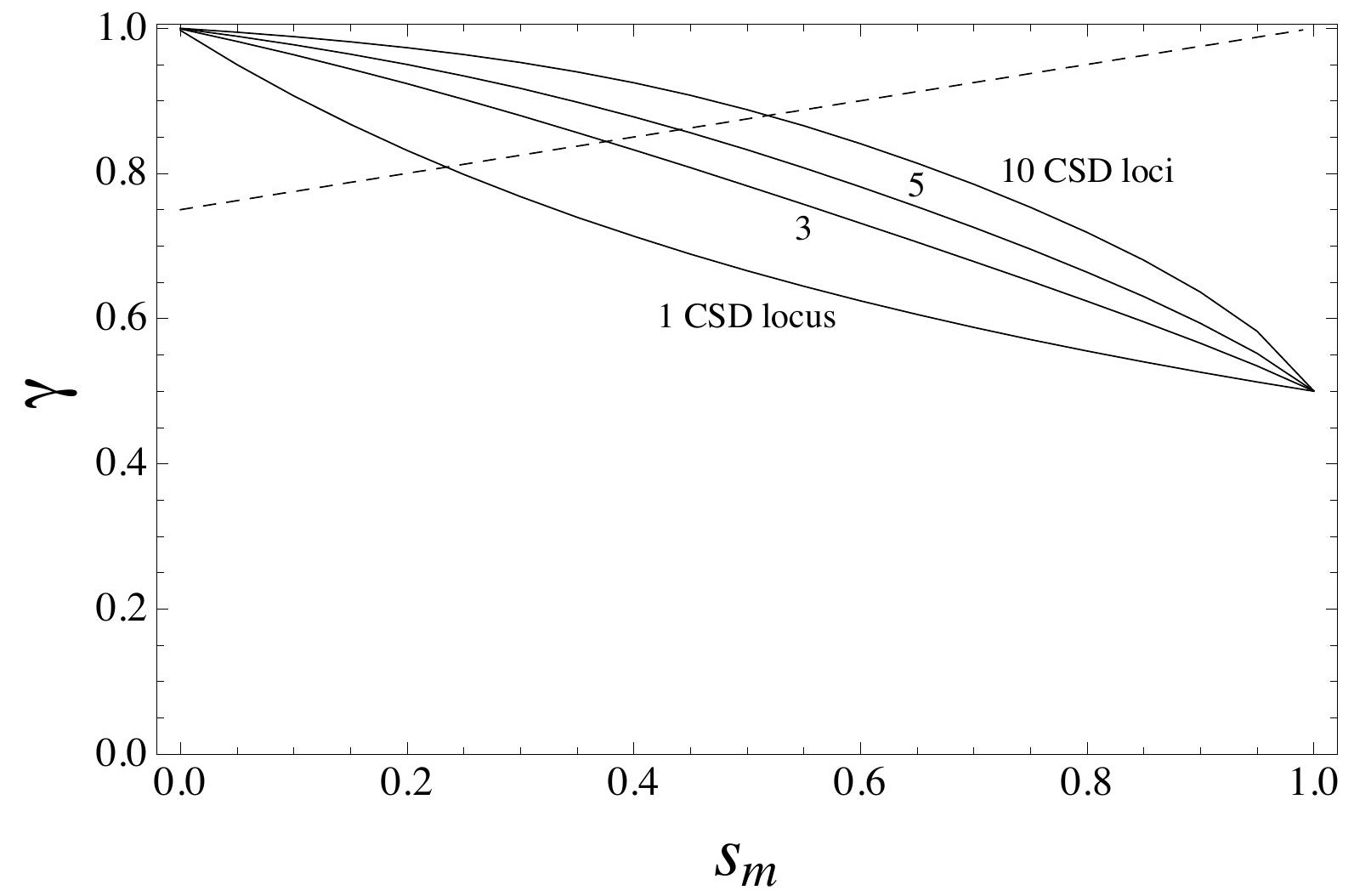


